\title{
ANALYSING $E_{k}\left|E_{r}\right| c$ QUEUES
}

Ivo J.B.F. Adan, Eindhoven University of Technology, NL

The $E_{k}\left|E_{r}\right| c$ queueing system is a typical example of a problem the analysis of which is more complex than the simple formulation suggests. The equilibrium equations become almost intractable if one attempts to solve these equations via multi-dimensional generating function techniques.

In this talk we show that the equilibrium distribution can be represented by a linear combination of geometric terms by using a direct approach (without generating functions). This approach is based on the separation of variables technique, familiar from the theory of partial differential equations, but which is less well known for partial difference equations. The essential idea is first to construct a sufficiently rich base of separable solutions of the equations in the interior of the state space and then to use this base to construct a linear combination that also satisfies the conditions at the boundaries of the state space. The separable solutions appear to be geometric in each of the state variables.

The representation of the equilibium distribution provides insight in the behaviour of the processes and it can directly be used for numerical calculations. 